\title{
Interactive comment on "Hydrodynamic
} characterization of past flash-flood events and their associated hazards from dendrogeomorphological evidence in Caldera de Taburiente National Park (Canary Islands, Spain)" by Julio Garrote et al.

Julio Garrote et al.

juliog@ucm.es

Received and published: 29 September 2016

First, the authors would like to thank the anonymous reviewer for his/her suggestions and comments, which have helped us improve our work. However, the authors disagree in some cases with the reviewer's comments and below we will attempt to respond to each issue.

1- I do not think that the statistical analysis of rainfall data is correct. I am surprised

Printer-friendly version

Discussion paper 
that the GEV and POT-GP method give such different results. In theory they should be analogous (being a Pareto distribution for peak-over-threshold mathematically correspondent to a GEV distribution for annual maxima). Is it because of the method used to estimate the parameters? I strongly recommend to add the plotting position representation of the data in figure 8 (which, by the way, should have rainfall and not flood peak in the y-axis). This would show where the estimation problem has gone wrong.

In the case of the statistical analysis of rainfall data presented in the paper, the GEV distribution was fitted using three different methods: moments, probability weighted moments, and maximum likelihood. The SQRT-ET max distribution was fitted using the maximum likelihood method and the GP-POT distribution was fitted using the Lmoments method. It is also worth mentioning that SQRT-ET max distribution (Etoh et al., 1986) was proposed by the Spanish Government in the manual "Maximum daily rains in continental Spain, (Ministry of Civil Works, 1999)", and has been used in numerous scientific studies conducted in Spain (for example, Ruiz-Bellet et al. 2015, Bianucci et al. 2013, Bianucci et al. 2015, or Rico et al. 2001). One of the reasons given in this manual for choosing the SQRT-ET max distribution (as opposed to others such as GEV, TCEV, and LP3) was that it provided more conservative results than other distributions. In our case, this claim showed to be true, with both functions providing similar values over a return period of 25 years and, beyond that, the SQRT- ET max distribution actually giving more conservative results. It is however true that this behavior does not occur when comparing the results shown by the GEV and SQRT-ET max distributions. From the above, and as the reviewer proposes, a full review of the statistical analysis conducted will be provided, with special emphasis on the results shown by the GEV distribution.

2- I do not agree with the strategy of using over sofisticated methods when order of magnitudes are of interest. For instance, in section 3.2.2 the method for modelling the routing of the flood wave is presented, which require LIDAR data for the channel morphology. This is coupled with a very crude ranoff generation model (SCS-CN method

Interactive comment
Printer-friendly version

Discussion paper 
+ unit hydrograph with parameters estimated from empirical formulae) forced by highly uncertain rainfall inputs. What is the rationale for doing this? Much better would be to use simple models and account for the uncertainties involved.

The authors also share the view that the estimation of uncertainties is an important aspect. However, we do not believe that the use of simpler models is much better. Therefore, our approach was to try to use the best possible models conditioned by the availability and quality of data. In short, where the input data allow, more sophisticated methods will be used to avoid the accumulation, at least in these processes, of errors and uncertainties. For example in the case of the hydraulic model, we consider the use of a high detailed DEM to represent as closely as possible the actual ground surface morphology, which improves the accuracy of the results. For this reason, and given the availability of data, we chose to use LIDAR topographic data within a $2 \mathrm{D}$ hydraulic model. The choice of this type of hydraulic model was based on its greater ability to reproduce the complex movement of water in these kinds of avenues. With regard to the rainfall-runoff model, we chose the simulation methods for the various processes involved based on the information available for the basin. The choice of the SCS-CN loss method, which is well-developed and widely accepted in the United States and abroad (Ponce and Hawkins, 1996), was based, on the one hand, on the availability of high resolution orthophotographs for the area and, on the other, on the possibility of conducting fieldwork to test and adjust the values previously obtained by photo-interpretation. Thus, input data concerning the $\mathrm{CN}$ value was considered as representative of the basin characteristics. The use of other methods of estimating initial losses or abstractions (like Green-Ampt) was discarded due to the lack of more detailed soil composition and textural data. Finally, regarding the transformation method (Clark Unit Hydrograph), the absence of rainfall and flow data associated to the same event reduces the availability of transformation methods in the HEC-HMS environment to only two options. With regard to the consideration of the associated uncertainties, although we considered this to be an essential point, the absence of flow data to calibrate and validate the hydrological output model flows makes it impossible to estimate

Interactive comment
Printer-friendly version

Discussion paper 
this in each hydrological calculation and its overall effect as the methodology unfolds.

3- I strongly disagree with the "Future prospect" section of the paper, which suggests to use more complex models in the future. This will add other uncertainties and will not solve those discussed within this paper.

In this case, the authors do not agree with the reviewer's opinion. The authors do not propose a move towards more complex models, but toward models that attempt to reflect the complexity of flash-flood processes. Undoubtedly, a degree of uncertainty exists for the results of any model with regard to the actual behavior of the modeled phenomenon. Efforts to make the starting point of the model as close to reality as possible should be one of the ultimate goals. The use of clear water along with a low-resolution DEM could be useful in the hydraulic modelling of the slow onset floods typically found in main rivers and in non-urban areas. However, for flash floods in both urban areas and small mountain basins, previous studies have shown that more simple hydraulic models do not work correctly. 1D hydraulic models are not able to solve the complexity of this type of floods, and 2D hydraulic models provide better results (for example: Costabile et al. 2015, Ernst et al. 2010, o Mignot et al. 2006). Clearly, further work is required to better understand the behavior of small, mountain basin flash floods in order to gradually reduce the uncertainties associated with each process. However, as shown in this work with the use of dendrogeomorphological evidence, these uncertainties can also be reduced by applying proxy data from floods that have occurred in the past.

4- The title and abstract state that a frequency analysis of (8?) past flash-flood events is performed. In reality only one event is analysed and the probability of exceeding such an event has not been estimated. The "Conclusion" section does not say what the paper contains.

Actually, in the Abstract the authors state that dendrogeomorphological data is available for eight different flood events. But later, in the same abstract, the authors focus

Printer-friendly version

Discussion paper 
the paper on the 1997 flood event. This discrepancy is due to the availability of sufficiently replicated and representative dendro evidence for the study. Thus, from the eight different flood events with dendrogeomorphological evidence, only the data from the 1997 event has a wide enough spatial distribution to be valid for comparison with the results of hydraulic modeling. In the other flood events, or the amount of dendro data was not enough (few replications) or the spatial distribution was highly skewed and, consequently, it was not considered valid for comparison with the results from the hydraulic models. Be that as it may, the Abstract will be reviewed to try to rectify any confusion in the interpretation of the analysis carried out.

5- The methodologies used have not been presented clearly. For instance, what is the "SQRT-ETMAX method"?

This is an extreme value distribution proposed by Etoh et al. (1986), which has been broadly used in Spain for annual maximum rainfall data statistical analysis. SQRT distribution has been used to calculate the daily maximum rainfall in Spain for different return periods (Spanish Civil Work Ministry, 1999). Several studies regarding SQRT distribution and its application in Spanish rainfall series have been done, for example: Ferrer, 1996; Zorraquino, 2000.

6- What is the "RMSE over disturbed tree sample"?

This value refers to the fit error between the heights of the nibs (dendrogeomorphological evidence, FDES) and the water surface height associated with flood modeling in that location.

7- What model has been used to generate synthetic hyetographs?

The synthetic hyetograph has been built using the alternating block method (Chow et al. 1994).

8- How have the daily precipitation observations been used to constrain the generated hourly timeseries?

Printer-friendly version

Discussion paper 
Distribution of daily rainfall in the form of hourly time series has been done using the IDF curves currently used in Spain for the study area (Salas and Fernandez, 2007). NHESSD The procedure for obtaining these IDF curves is described in works like Salas and Fernandez-Yuste (2006a and 2006b).

Interactive comment on Nat. Hazards Earth Syst. Sci. Discuss., doi:10.5194/nhess-2016-206, Interactive 2016.

comment

Printer-friendly version

Discussion paper 